\title{
Genotoxic Impurities and Its Risk Assessment in Drug Compounds
}

\author{
Amit Gosar $^{1}$, Hussain Sayyed ${ }^{2}$ and Tabrez Shaikh ${ }^{3 *}$ \\ 1,3Indoco Research Centre, Navimumbai, Maharashtra, India \\ ${ }^{2,3}$ Sir Sayyed College, Department of Chemistry, Aurangabad, India
}

Received: 州 October 17, 2018; Published: 跳 October 23, 2018

*Corresponding author: Tabrez Shaikh, Indoco Research Centre, Rabale, Navi Mumbai, Maharashtra, India

\begin{abstract}
The present work is based on a assess analysis of the literature in order to define the genotoxic impurities present in the drug substances and drug products. Starting from the definition of genotoxic impurities, sources of genotoxic impurities, their generation and classification based on their toxicity. The article also explains the various regulatory guidelines to control the genotoxic impurities in drug substances. Isolation, qualification of impurity and its identification along with quantification by the various analytical methods is also described in this review article.
\end{abstract}

Keywords: Impurities; Genotoxic; Classification; Guidelines; Drug substances

\section{Introduction}

Pharmaceutical drugs are always associated with some unwanted chemicals along with active components. This unwanted chemical compound is called an impurity. Thus, the common definition of impurity is any substance coexisting with the drug substance, such as starting material, reagents, catalyst, raw material or intermediates arising during the synthesis or develop during storage or shipment of the drug. Impurities provide no benefit to the patient while it may have the potential to cause the adverse effect. International Council for Harmonisation in its guideline ICH S2 (R1) defines genotoxicity as "a broad term that refers to any deleterious change in the genetic material, regardless of the mechanism by which the change is induced." While genotoxic impurities have been defined as "Impurity that has been demonstrated to be genotoxic in an appropriate genotoxicity test model, e.g., bacterial gene mutation (Ames) test" [1]. A potential genotoxic impurity (PGI) has been defined as an "Impurity that shows a Structural alert for genotoxicity but that has not been tested in an experimental test model. Here potentially relates to genotoxicity, not to the presence or absence of this impurity". Genotoxic impurities impact the genetic material by means of mutations through chromosomal breaks, rearrangements, covalent binding or insertion into the DNA during replication. These changes in the genetic material, caused by the exposure to very low levels of a genotoxic chemical, can lead to cancer. Thus, it is very important to identify genotoxic impurities in drugs followed by monitoring and control at very low levels to ensure safety to the public $[2,3]$.

\section{Sources of Genotoxic Impurities}

Genotoxic impurities can get incorporated into drug substances through the various sources, the major source is the starting material used in the synthesis of drug substances and its impurities. Similarly, genotoxic intermediate and by-products formed in the synthesis process may get be carried forward to the drug substances as genotoxic impurities. Besides these, solvents, catalyst and reagent used in the synthesis process can also be a potential source of genotoxic impurities in drug substances. Degradation product generated on storage and shipment or on exposure to light, air oxidation or hydrolysis contribute to the generation of impurities in drug substances. If drug substance required is a specific isomer then stereoisomers of raw material and intermediate also contribute to the generation of chiral impurities in drug substances. Figure 1 shows the formation of impurities in various stages of drug synthesis. Beside these excipients and their impurities, extractable and leachable can also contribute to genotoxic impurities in drug products $[4,5]$ (Table 1$)$. 


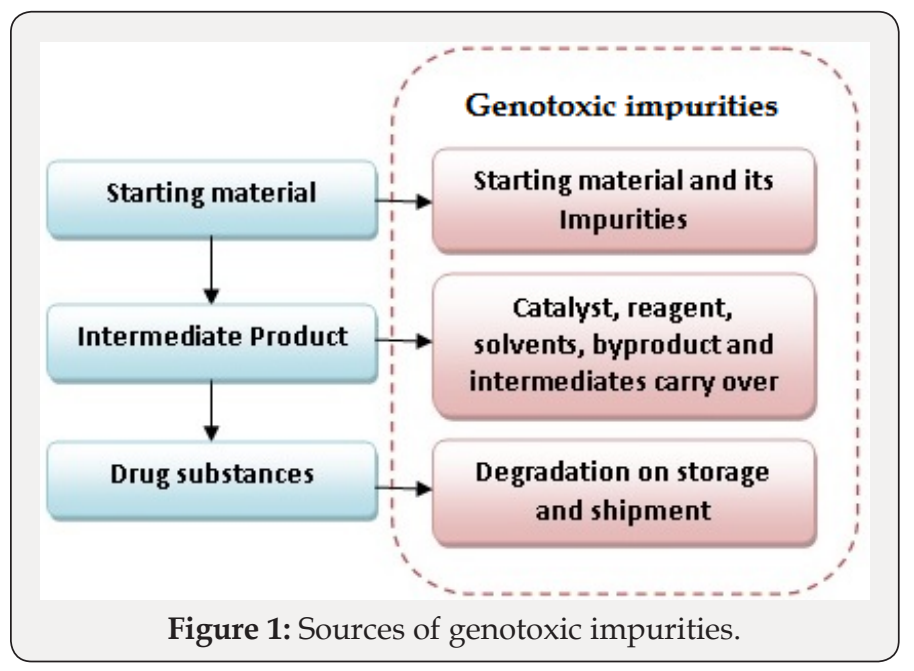

Table 1: Genotoxic compounds in drug substances.

\begin{tabular}{|c|c|}
\hline Category/Stage & Compounds \\
\hline Starting material & Hydrazine, Nitroso, acrylonitrile compounds \\
\hline Intermediate & Benzaldehyde, Nitro compounds \\
\hline By-product & Sulphonate esters, phosgene \\
\hline Reagent & $\begin{array}{c}\text { Formaldehyde, epoxides, esters of phosphate \& } \\
\text { sulphonates }\end{array}$ \\
\hline Solvent & Benzene, 1,2-dichloroethane \\
\hline Catalyst & Toxic heavy metals, metal phosphates \\
\hline Degradation product & N-oxides, aldehydes, \\
\hline
\end{tabular}

\section{Regulatory Guidelines}

\section{Pharmaceutical Research and Manufacturers Association (PhRMA)}

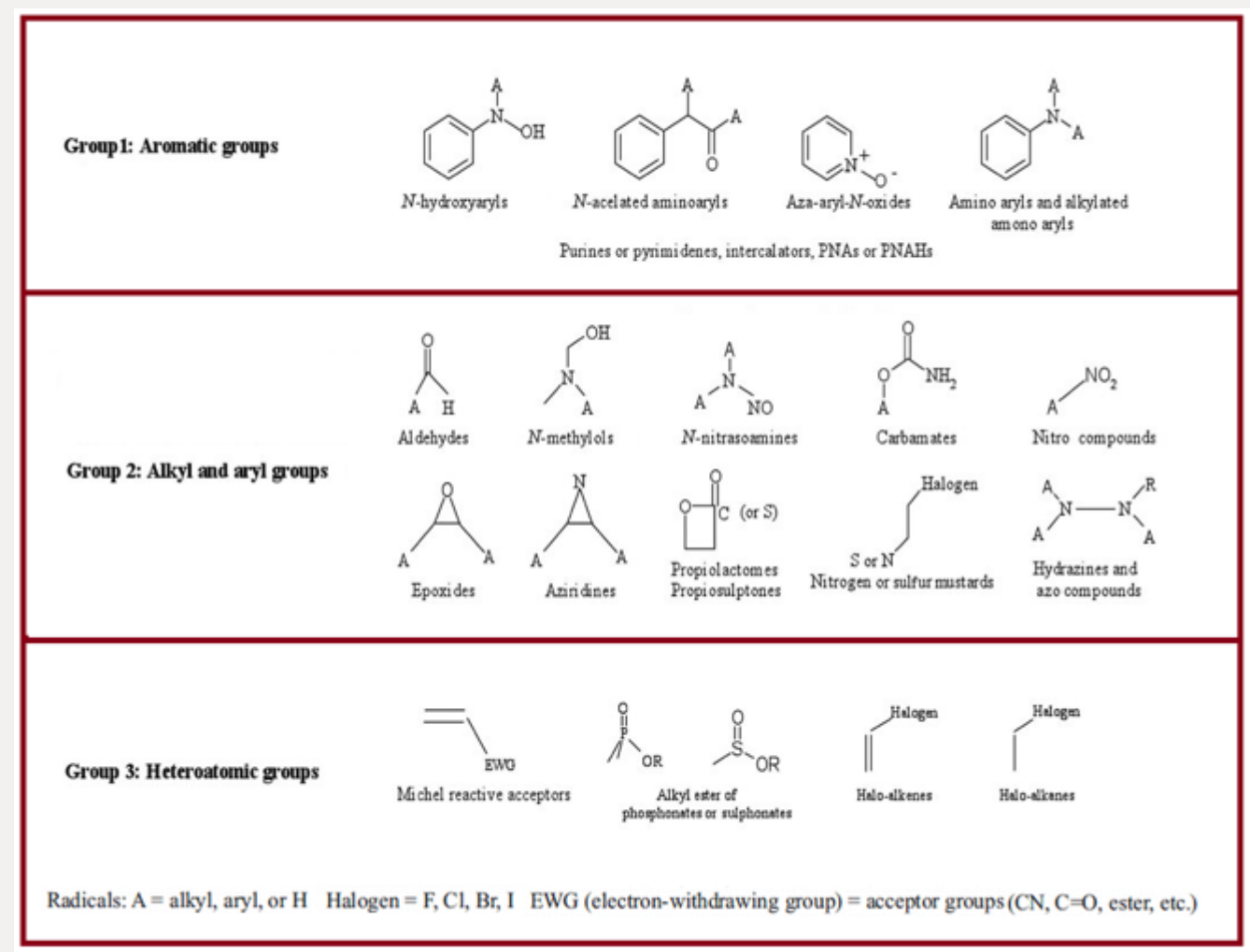

Figure 2: Structure alerting functional groups.

The PhRMA published a procedure for the testing, classification, qualification, and toxicological risk assessment of genotoxic impurities in 2006. They listed genotoxic impurities in the different group based on their functional groups involved which react with DNA. These functional groups were categorized into three groups such as Aromatic, Alkyl /Aryl and Hetero atomic (Figure 2) [6].

\section{European Medicine Agency (EMA)}

EMA introduced the Guideline on the Limits of Genotoxic Impurities after the draft releases for consultation in 2002 and
2004 with the final version released in 2006. The toxicity data available for genotoxic impurities is quite variable and it is the only main factor that governs the process used for the assessment of acceptable limits. In the absence of data, there is usually the need for one established risk assessment method. Therefore, EMA firstly introduced the concept and values for the threshold of toxicological concern (TTC) to control the genotoxic impurities. EMA also introduced questions and answers on the Guideline on the Limits of Genotoxic Impurities which was published in 2010 for clarification and harmonization of genotoxicity guideline [7]. 


\section{Food Drug Administration (FDA)}

USFDA published a draft guidance for industry entitled Genotoxic and Carcinogenic Impurities in Drug Substances and Products in Dec. 2008. The FDA guidance provides specific recommendations regarding the safety qualification of impurities with known or suspected genotoxic or carcinogenic potential. The guidance describes a variety of ways to characterize and reduce the potential cancer risk with patient exposure to genotoxins and carcinogenic impurities. The approaches were similar to the EMA guideline [8] (Table 2).

Table 2: FDA guidance steps risk minimization.

\begin{tabular}{|c|c|}
\hline Step & Action \\
\hline 1 & $\begin{array}{c}\text { Change the synthesis or purification process to minimize the } \\
\text { formation and maximize the removal of the relevant impurity }\end{array}$ \\
\hline 2 & $\begin{array}{r}\text { Allow a maximum daily exposure target of } 1.5 \mu \mathrm{g} \text { per day of the } \\
\text { relevant impurity as a general target. }\end{array}$ \\
\hline 3 & $\begin{array}{c}\text { Further characterize the genotoxic and carcinogenic risk to } \\
\text { better support appropriate impurity specifications, either for } \\
\text { higher or lower values }\end{array}$ \\
\hline
\end{tabular}

\section{International Council for Harmonisation (ICH)}

The first guideline related to genotoxicity was introduced by ICH in July 1995 as S2A: Guidance on Specific Aspects of Regulatory Genotoxicity Tests for Pharmaceuticals; this guideline provided specific guidance and recommendations for in vitro and in vivo tests and on the evaluation of test results. The second guideline was in 1997 as S2B: Genotoxicity: A Standard Battery for Genotoxicity Testing for Pharmaceuticals; In 2013 M7 guideline was published which offer guidance on the analysis of Structure-Activity Relationships (SAR) for genotoxicity. After which M7 (R1) guideline was released in step 2 in June 2015 and step 4 in May 2017. The M7 (R2) is undertaken for the revision. This guideline incorporates acceptable limits (Acceptable Intakes (AIs) or Permitted Daily Exposures (PDEs)) for new DNA reactive (mutagenic) impurities and revising acceptable limits for impurities already listed in the Addendum as new data becomes available, which will result in the future ICH M7 (R2) version [9,10].

\section{Classification of Genotoxic Impurities}

Genotoxic impurities are classified based on their risk assessment involves an initial analysis of actual and potential impurities by conducting database and literature searches for carcinogenicity and bacterial mutagenicity data which classify them to Class 1, 2, or 5. If data for such a classification is not available, an assessment of Structure-Activity Relationships (SAR) that focuses on bacterial mutagenicity predictions is performed. This could classify it into Class 3, 4, or 5. Each class is defined as below [10].

Class 1: These impurities have established mutagenic and carcinogenic data and are known to be the most serious risk and need to eliminate them by modifying the process. If this is not possible, these impurities are to be limited at "Threshold of Toxicological Concern (TTC)" as the last option.

Class 2: These impurities have the well-established mutagenic data, but their potential to cause carcinogen is not known. Hence, these impurities need to be controlled using the TTC approach.

Class 3: These impurities are having alert structures unrelated to the structure of the drug substances and of unknown genotoxic potential. Based on functional groups within their molecule, they can be classified as genotoxic. The toxicity of these impurities is identified based on the structure-activity relationship (SAR).

Class 4: These impurities are having structures similar to the structure of drug substances and additionally contain functional or moiety that has potentially alert shared with the parent structure and consider to be non-genotoxic.

Class 5: These impurities have no alert structures, and evidence indicates the absence of genotoxicity. These compounds are to be treated as normal impurities and controlled according to the ICH guidelines.

\section{Threshold of Toxicological Concern (TTC) Approach}

As toxicological assessment of all the impurities is very difficult, therefore the threshold of toxicological concern (TTC) was proposed by EMEA (2006) to control the genotoxic impurities. Where acceptable intake of a mutagenic impurity of $1.5 \mu \mathrm{g}$ per person per day is considered to be associated with a negligible risk (theoretical excess cancer risk of $<1$ in 100,000 over a lifetime of exposure). This approach would usually be used for mutagenic impurities present in pharmaceuticals for long-term treatment greater than 10 years and where no carcinogenicity data are available (Classes 2 and 3). The limit for individual genotoxic impurity can be calculated by the formula given below [11].

Limit $(\mathrm{ppm})=1.5(\mu \mathrm{g} /$ day $) /$ maximum daily dose $(\mathrm{g} /$ day $)$

Considering all the drug are not used for long-term treatment, the new concept staged TTC was introduced by Mueller. This concept takes into account the fact that the duration of exposure is a key factor impacting on the probability of a carcinogenic response. The recommended limits for daily intake of genotoxic impurities are as given in Table 3 .

Table 3: Staged TTC daily intake.

\begin{tabular}{|c|c|c|c|c|c|}
\hline \multirow{2}{*}{} & \multicolumn{5}{|c|}{ Duration of exposure } \\
\cline { 2 - 6 } & $\begin{array}{c}\text { Single } \\
\text { dose }\end{array}$ & $\begin{array}{c}\leq 1 \\
\text { month }\end{array}$ & $\begin{array}{c}\leq 3 \\
\text { months }\end{array}$ & $\begin{array}{c}\leq 6 \\
\text { months }\end{array}$ & $\begin{array}{c}\leq 12 \\
\text { months }\end{array}$ \\
\hline $\begin{array}{c}\text { Allowable daily } \\
\text { intake }\end{array}$ & $120 \mu \mathrm{g}$ & $60 \mu \mathrm{g}$ & $20 \mu \mathrm{g}$ & $10 \mu \mathrm{g}$ & $5 \mu \mathrm{g}$ \\
\hline
\end{tabular}




\section{Risk Assessment of Impurity}

The control of genotoxic impurities (GTIs) is a very important activity that is performed for any drug substances or drug product. A key element of this is the risk assessment. Primary components of such an assessment are to focus specifically on the effective use of in silico assessment tools. For this impurity, the structure is first identified and conducted GTI assessment. If impurity structure shows no alert, then an impurity is controlled as a normal impurity as per ICH Q3 guidelines. But if impurity shows structure alert, then it is subjected to Ames test. A negative result for Ames test leads to control impurity as per ICH Q3 guideline. If Ames test is positive, then an impurity is limited to safety level as per the TTC approach. Knowledge of the chemical structure of impurity and its formation mechanism is very important to assess its toxicological implications thus improving the synthetic chemical processes to reduce or eliminate the impurity [11] (Figure 3).

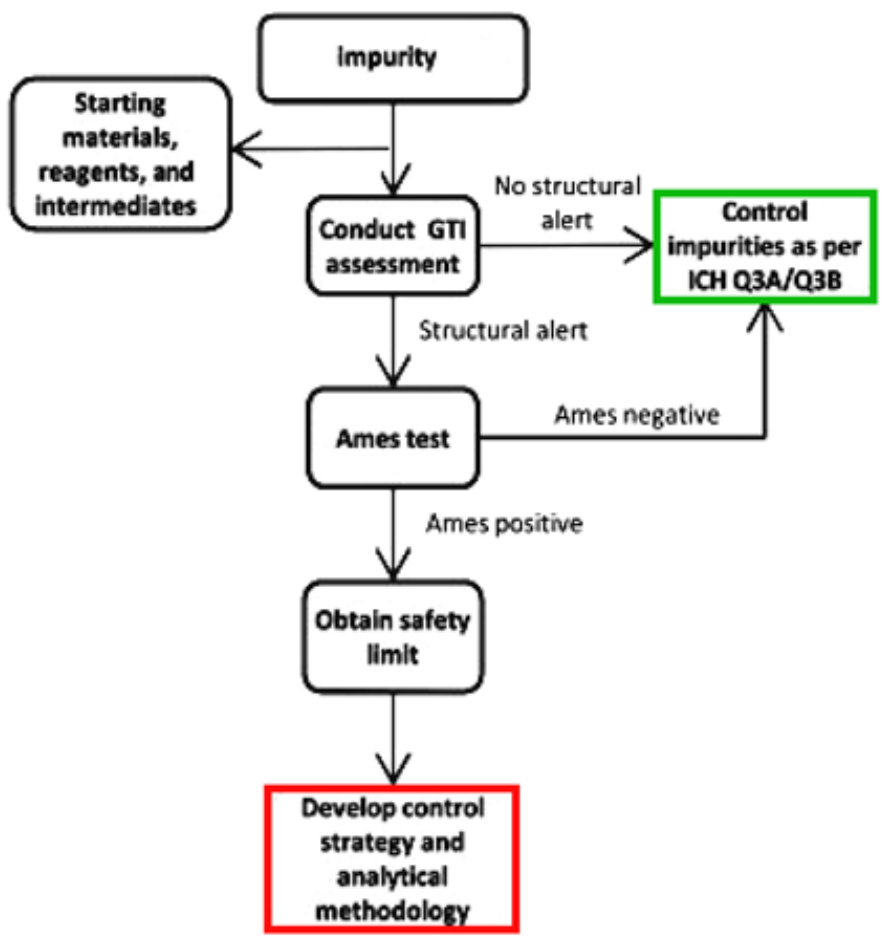

Figure 3: Genotoxic impurity assessment decision tree.

\section{Isolation of Genotoxic Impurities}

For isolation of the impurities, mostly the chromatographic techniques are used along with classical. Isolation of impurities is very necessary to establish its structure and toxicity. The following methods have been used for isolation of genotoxic impurities [12].
a) Solid-phase extraction
b) Column chromatography
c) Flash chromatography
d) Supercritical fluid chromatography
e) Thin-layer chromatography
f) Capillary electrophoresis
g) Preparative high-pressure liquid chromatography
h) Accelerated solvent extraction

i) Liquid-liquid extraction

Isolation of impurities should be initially based on simple extraction or partition methods. It may be possible to extract impurities selectively on the basis of acidity, basicity, or neutrality. The extraction process usually involves liquid-liquid extraction, where one phase is an aqueous solution and the other is an organic phase that is nonpolar. In chromatographic method desired impurity peak or band is separated, concentrated, and isolated. This can be done using a simple chromatographic column prepared in the laboratory or by means of an instrument such as the Flash and a Preparative chromatograph [12].

\section{Analysis of Impurities}

\section{Identification of Impurity}

It is one of the activities of Impurity profiling, where the goal is to identify the chemical structures of impurities present in the drug substances or observed in the stability studies above a particular threshold. 
Identification of pharmaceutical impurities can be done by various spectroscopic techniques, such as Ultraviolet (UV), Infrared (IR) Mass (MS) and Nuclear magnetic resonance (NMR) while its quantitation can be done by chromatographic technique such as High performance liquid chromatography (HPLC) equipped with mass detector of UV detector, Gas chromatography (GC) equipped with mass, FID or ECD detectors, Supercritical fluid chromatography (SFC) and Thin layer chromatography (TLC/HPTLC). ICH guidelines indicate that all the genotoxic impurity present in drug substance should be identified if present at or above a certain limit which is called as identification threshold [13].

\section{Analytical Method Development}

The basic task for the development of an analytical method for genotoxic impurities is to develop the method which can detect the genotoxic impurities at trace levels and well below the TTC. The developed analytical method should have less variability by conducting a series of controlled experiments thus to make quality and safe drug products. As global regulatory requirements have gone more stringent, analytical methods for global products must be able to meet global regulatory requirements. Method development is a continuous process where the goal is to consistently improve the quality of the product [14].

\section{Analytical Method Validation}

A very general definition of validation is establishing documented evidence which provides a high degree of assurance that a specific procedure, process, equipment, activity or system will consistently produce a product meeting its predetermined specifications and quality attributes. Validation is an important feature after the development of any analytical method because it is closely related to the quality of the results. All analytical methods, whether qualitative or quantitative are required to be validated. The degree of validation varies for the type of method and its application. For several years now, method validation studies, guidelines and procedures have focused mainly on quantitative methods of analysis. Validation is an imperative activity in the process of impurities profiling where the developed analytical method used for the determination of genotoxic impurities in drug substances is validated in order to establish that the method is suitable for its aimed purpose. The analytical methods are validated with specificity, linearity, precision, accuracy, ruggedness, robustness and forced degradation parameters in accordance with ICH Harmonized Tripartite Guidelines [15,16].

\section{Conclusion}

The present review describes the details regarding genotoxic impurities in drug substances. The article also provides valuable information regarding the classification of impurities and regulatory guidelines to control these impurities. Various techniques used in the isolation of impurities and analytical techniques require for their determination and quantification along with analytical method development and its validation.

\section{References}

1. ICH S2 (R1) (2011) "The tripartite harmonised ICH Guideline S2 (R1): guidance on genotoxicity testing and data interpretation for pharmaceuticals intended for human use.

2. Custer LL, KS Sweder (2008) The role of genetic toxicology in drug discovery and optimization. Current drug metabolism 9(9): 978-985.

3. Yadav Usha, Priyanka Dhiman, Neelam Malik, Anurag Khatkar, Neelam Redhu, et al. (2013) Genotoxic impurities-an overview. Journal of Biomedical and Pharmaceutical Research 2(5): 39-47.

4. Szekely Gyorgy, Miriam C Amores de Sousa, Marco Gil, Frederico Castelo Ferreira, William Heggie (2015) Genotoxic impurities in pharmaceutical manufacturing: sources, regulations, and mitigation. Chemical reviews 115(16): 8182-8229.

5. Robinson Derek I (2010) Control of genotoxic impurities in active pharmaceutical ingredients: a review and perspective. Organic Process Research \& Development 14(4): 946-959.

6. Jacobson-Kram David, Timothy McGovern (2007) Toxicological overview of impurities in pharmaceutical products. Advanced drug delivery reviews 59(1): 38-42.

7. Committee for Medicinal Products for Human Use (2006) Guideline on the limits of genotoxic impurities. European Medicines Agency (EMEA), London UK.

8. (2008) Genotoxic and Carcinogenic Impurities in Drug Substances and Products. US Department of Health and Human Services, Rockville, MD.

9. Guideline, ICH Harmonised Tripartite (1995) Guidance on Specific Aspects of Regulatory Genotoxicity Tests for Pharmaceuticals S2A.

10. International Conference on Harmonisation (ICH) (2017) Assessment and control of DNA reactive (mutagenic) impurities in pharmaceuticals to limit potential carcinogenic risk, M7.

11. Kroes Robert, J Kleiner, A Renwick (2005) The threshold of toxicological concern concept in risk assessment." Toxicological sciences 86(2): 226230.

12. Ahuja Satinder, Karen Mills Alsante Eds. (2003) Handbook of isolation and characterization of impurities in pharmaceuticals, Vol. 5 (1 $\left.1^{\text {st }} \mathrm{Edn}\right)$ Academic press, USA.

13. Ahuja Satinder, Stephen Scypinski Eds (2010) Handbook of modern pharmaceutical analysis, Vol. 10 ( $2^{\text {nd }}$ Edn) Academic press, USA.

14. Kazakevich Yuri V, Rosario Lobrutto (2006) HPLC for pharmaceutical scientists. John Wiley \& Sons, USA.

15. Guideline, ICH Harmonised Tripartite (2005) Validation of analytical procedures: text and methodology Q2 (R1). International Conference on Harmonization, Geneva, Switzerland, pp. 1-13.

16. Chan Chung Chow, YC Lee, Herman Lam, Xue-Ming Zhang (2004) Analytical method validation and instrument performance verification. John Wiley \& Sons, USA. 


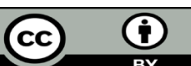

This work is licensed under Creative Commons Attribution 4.0 License

Submission Link:

Submit Article

DOI: 10.32474/DDIPIJ.2018.02.000143

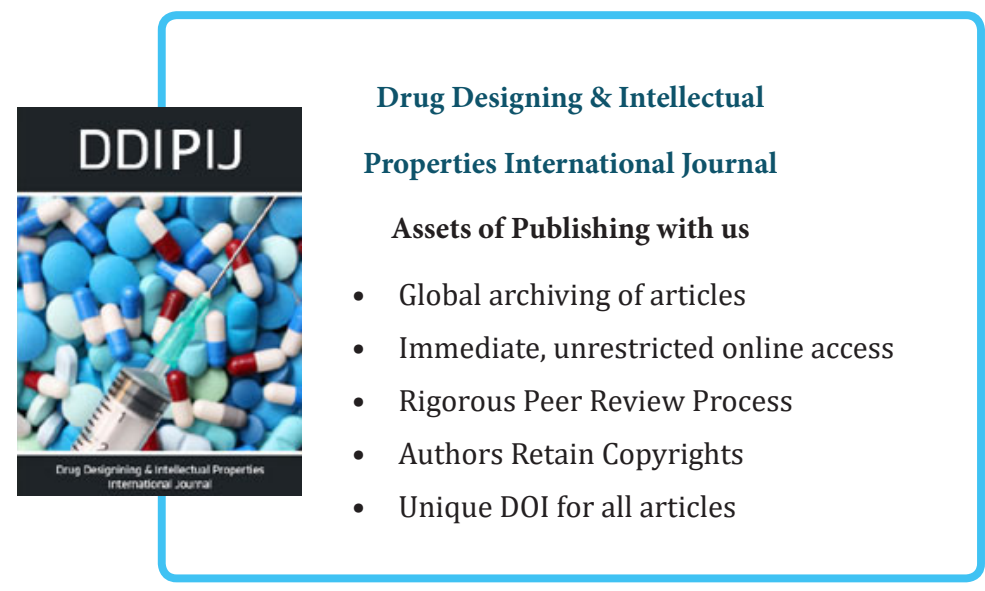

